\title{
SYNTHESIS AND PROPERTIES OF NEW FAMILY OF SUPERCONDUCTING COPPER OXIDES BASED ON GaO LAYERS*
}

ANL/CP- -74583

\section{B. Dabrowski, V. Zhang-McCoy}

DE92 005219

Department of Physics, Northern Illinois University, DeKalb, IL 60115

\section{P. Radaelli, A.W. Mitchell, D.G. Hinks}

Materials Science Division and Science \& Technology Center for Superconductivity

Argonne National Laboratory, Argonne, IL 60439

\author{
J.T. Vaughey, D.A. Groenke, K.R. Poeppelmeier
}

Department of Chemistry and Science \& Technology Center for Superconductivity

Northwestern University, Evanston, IL 60209

5th Annual Conference on Superconductivity and Applications, September 24-25, 1991, Buffalo, NY

imc

\section{DISCLAIMER}

\begin{abstract}
This report was prepared as an account of work sponsored by an agency of the United States Government. Neither the United States Government nor any agency thereof, nor any of their - mployees, makes any warranty, express or implied, or assumes any legal liability or responsiun'ity for the accuracy, completeness, or usefulness of any information, apparatus, product, or process disclosed, or represents that its use would not infringe privately owned rights. Reference herein to any specific commercial product, process, or service by trade name, trademark, manufacturer, or otherwise does not necessarily constitute or imply its endorsement, recommendation, or favoring by the United States Government or any agency thereof. The views and opinions of authors expressed herein do not necessarily state or reflect those of the United States Government or any agency thereof.
\end{abstract}

\footnotetext{
"Work supported by the U.S. Department of Energy, BES-Materials Sciences under contract \#W-31-109-ENG-38 (BD, AWM, DGH) and the National Science Foundation, Division of Materials Research - Low Temperature Physics Program under grant \#DNR 87.19738 (VZ) and the Science and Technology Center for Superconductivity under grant DMR88-09854 (PR, JTV, DAG, KRP).
}

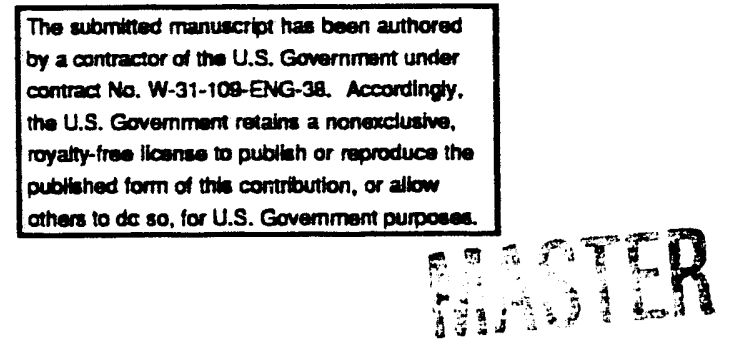


Submitted to: Fifth Annual Conference on superconouctionity and Applicutions, Sept. 24-26,1991, Buffalo, NY.

\title{
SYNTHESIS AND PROPERTIES OF NEW FAMILY OF SUPERCONDUCTING COPPER OXIDES BASED ON GaO LAYERS
}

B. Dabrowski and V. Zhang-McCoy, Department of Physics, Northern Illinois University, Deka1b, IL 60115

\author{
P. Radaelli, A.W. Mitchell and D.G. Hinks, \\ Materials Science Division, Argonne National Laboratory, \\ Argonne, IL 60439
}

J.T. Vaughey, Department of

D.A. Groenke and K.R. Poeppelmeier, Chemistry, Nor: hwestern University, Evanston, IL 60208

\section{ABSTRACT}

We have discovered the first layered superconducting copper oxide with small, fixed oxidation state cations separating the conducting $\mathrm{CuO}_{2}$ planes. This material, $\operatorname{GaSr}_{2} \mathrm{Y}_{1-x} \mathrm{Ca}_{x} \mathrm{Cu}_{2} \mathrm{O}_{7}$, is similar to $\mathrm{YBa}_{2} \mathrm{Cu}_{3} \mathrm{O}_{7}$ with the square planar copper chains replaced by chains of edgeshared $\mathrm{GaO}_{4}$ tetrahedra. Thus, oxidation can occur only for the copper ion located in square pyramidal coordination in the $\mathrm{CuO}_{2}$ plane. The undoped parent compound, $x=0$, does not show magnetic order above $4 \mathrm{~K}$, probably due to the presence of the thick, ionic reg.on separating the $\mathrm{CuO}_{2}$ planes. However, this ionic region does not suppresses high $T_{C}$ superconductivity ( 70K) for the doped compositions.

\section{INTRODUCTION}

All known copper oxide superconductors have an anisotropic structure containing two-dimensional $\mathrm{CuO}_{2}$ planes with the copper ion in square planar, square pyramidal or octahedral coordination by oxygen. The $\mathrm{CuO}_{2}$ planes are bounded in the third dimension by metaloxygen layers, AO, containing large ard strongly electropositive ( $\mathrm{A}=\mathrm{Ba}, \mathrm{Sr}$ and $\mathrm{La}-\mathrm{Ga}$ ) metal ions, thus forming $\mathrm{AO}-\mathrm{CuO}_{2}-\mathrm{AO}$ structural blocks. For many superconducting compounds there are frequently multiple $\mathrm{CuO}_{2}$ planes, separated by metal layers, $A^{\prime}\left(A^{\prime}=\mathrm{Ca}\right.$, lanthanides and $Y)$, within the block. All superconducting compounds can be divided into two general śtructural classes depending on the block stacking sequence. For the first class, blocks are 
stacked directly together (e.g. $\mathrm{La}_{2} \mathrm{CuO}_{4}, \mathrm{Nd}_{2} \mathrm{CuO}_{4}$ and $\mathrm{La}_{2} \mathrm{CaCu}_{2} \mathrm{O}_{6}$ ) [1]. The second class, contains an additional intermediate region, separating the blocks, consisting of mixed oxidation state cations covalently bonded to oxygen (e.g. structures based on $\mathrm{Cu}, \mathrm{Tl}, \mathrm{Bb}$ and $\mathrm{Bi}$ ) [1].

The best known superconducting copper oxide InBa ${ }_{2} \mathrm{Cu}_{3} \mathrm{O}_{7}$ ( $\mathrm{In}=$ lanthanides and $\mathrm{Y}$ ) contains square planar coordinated copper ions in the intermediate region (frequently referred to as the chain region) between the $\mathrm{AO}-\mathrm{CuO}_{2}-\mathrm{A}^{\prime}-\mathrm{CuO}_{2}-\mathrm{AO}$ blocks[2]. It was shown for $\mathrm{Ln}_{n}=$ La that it is possible to substitute this square planar copper site with tantalum and niobium resulting in the distinct, but very similar $\mathrm{LaBa}_{2} \mathrm{TaCu}_{2} \mathrm{O}_{8}$ structure, with octahedraly coordinated $\mathrm{Nb}$ and $\mathrm{Ta}[3]$. This latter structure shows the importance of the coordination preference of small ions leading either to the formation of ordered $\mathrm{CuO}_{2}$ planar compounds or mixing with copper on both sites. Recent discussion of the ionic size and coordination factors that led to the formation of $\mathrm{CuO}_{2}$ planes in $\mathrm{AA}^{\prime} \mathrm{BCuO}_{6}$ ( $\mathrm{B}=$ transition or post transition metal) compounds is given by Anderson et al. [4]. Several new layered copper oxides with ionic, fixed oxidation state, cations in the intermediate region between $\mathrm{AO}-\mathrm{CuO}_{2}-\mathrm{AO}$ structural blocks were found (e.g. single $\mathrm{CuO}_{2}$ layer $\mathrm{AlSrLaCuO}_{5}, \mathrm{GaSrLaCuO}_{5}$ and SnLa ${ }_{2} \mathrm{CuO}_{6}$, and double $\mathrm{CuO}_{2}$ layer $\mathrm{AlSr}_{2} \mathrm{LnCu}_{2} \mathrm{O}_{7}$ and $\left.\mathrm{GaSr}_{2} \mathrm{InCu}_{2} \mathrm{O}_{7}\right)$. However, none of these compounds was superconducting when prepared in air.

The structural similarity of these compounds to the known hole-doped copper oxide superconductors and the possibility of controlling the carrier concentration by varying the oxygen content led is to synthesize the Casubstituted compositions under high oxygen pressure. The combined calcium and oxygen doping could introduce the necessary charge to the $\mathrm{CuO}_{2}$ layers. Recently, we have

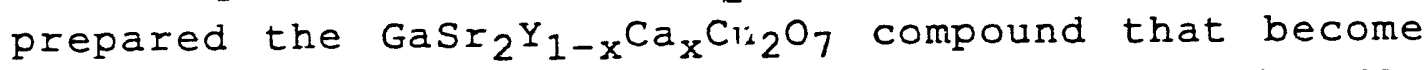
superconducting after high pressure oxygen annealing[5]. While the undoped parent compound, $x=0$, did not show three-dimensional magnetic order above $4 \mathrm{~K}$, probably due to the presence of the thick ionic region separating the $\mathrm{CuO}_{2}$ layers, superconductivity with a high $\mathrm{T}_{\mathrm{C}}(\sim 7 \mathrm{OK})$ was observed for the doped compositions.

\section{EXPERIMENTAL}

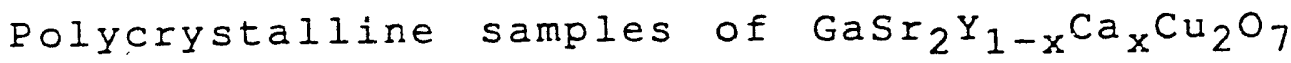
$(0 \leq x \leq 0.4)$ were synthesized from stoichiometric mixture 
of oxides and carbonates in air at $980^{\circ} \mathrm{C}$ followed by fast cooling to room temperature. Samples were fired for 3 weeks with frequont intermediate grindings. High pressure annealing was done for 24 hours in pure oxygen using $200 \mathrm{~atm}$. at $910^{\circ} \mathrm{C}$ for powdered samples and 300 atm. at $925^{\circ} \mathrm{C}$ for pressed dense pellets. Lattice parameters were determined using Rietveld refinement of powder $x$-ray diffraction data. Susceptibility measurements were performed using a squid (Quantum Design Corp. MPMS) and an a.c. (Lake Shore Cryotronics) susceptometer. Resistivity was measured using a standard four-lead d.c. technique. Oxygen content was determined using thermogravimetric analysis measurements (Cahn TG 171).

\section{RESULTS}

The orthorhombic structure of $\mathrm{GaSr}_{2} \mathrm{InCu}_{2} \mathrm{O}_{7}$ (noncentrosymmetric space group Ima2, No.46) is similar to $\mathrm{InBa}_{2} \mathrm{Cu}_{3} \mathrm{O}_{7}$ (see $\mathrm{Fig.1}$ ) $[5,6]$. The square planar copper chains in $\mathrm{LnBa}_{2} \mathrm{Cu}_{3} \mathrm{O}_{7}$ are replaced by chains of edgeshared $\mathrm{GaO}_{4}$ tetrahedra. The large lanthanides and $\mathrm{Sr}$ are distributed over the $A$ and $A^{\prime}$-cation sites and the small lanthanides occupy only the $A^{\prime}$ site between the copper planes within the double $\mathrm{CuO}_{2}$ layer.

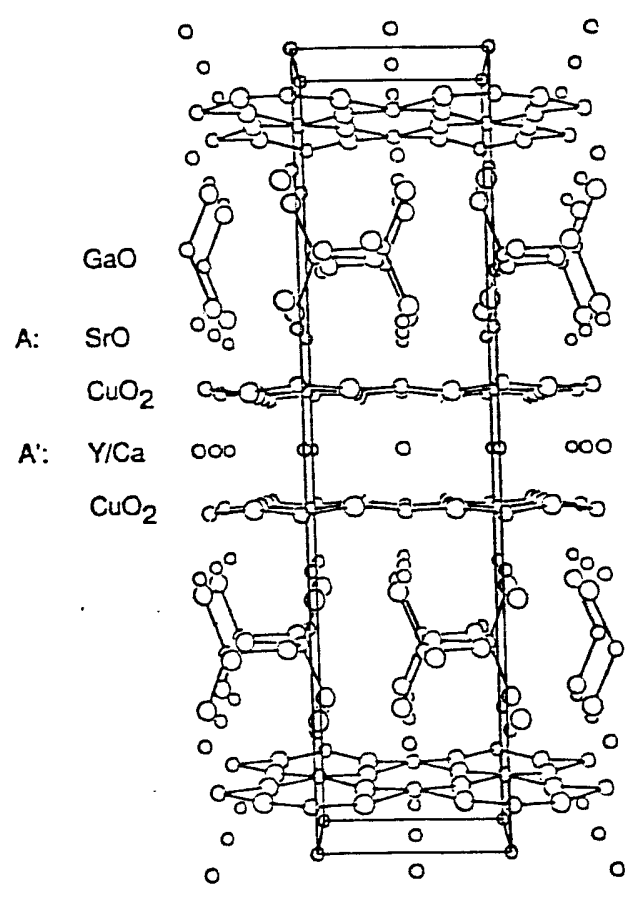

Fig.1 Layered structure of $\mathrm{GaSr}_{2} \mathrm{LnCu}_{2} \mathrm{O}_{7}$ viewed along the $b$ axis. 
Air cooled samples with compositions $0 \leq x \leq 0.25$ were single phase. Larger doping levels led to the presence of small amounts of unidentified impurity phases. In general, a very small contraction of the in-plane and an expansion of the out-of-plane lattice parameters were observed. with increasing doping. However; these lattice parameters changes were not continuous which indicates that the oxygen content and the cation distribution of the fast cooled samples may vary. The high pressure annealed samples showed a decreased amount of impurity phase for $x \geq 0.25$ and noticeable contraction of the inplane lattice parameters, indicating an increased holedoping of the $\mathrm{CuO}_{2}$ planes.

High sensitivity, zero field cooled squid susceptibility measurements using 100 Gauss were done for both powder and pellet high pressure annealed samples. For the dense pellets, a gradual development of a superconducting phase with an almost fixed transition temperature, $T_{C} \sim 20-25 \mathrm{~K}$, was observed with increased doping as shown on Fig. 2. For powder annealed samples different $\mathrm{T}_{C}$ 's were observed with the highest $\mathrm{T}_{C}=73 \mathrm{~K}$ for $x=0.3$ (see insert to Fig.2). The superconducting phase fractions, as determined from these measurements, were only a few percent. The squid measurements performed for the fast cooled undoped material over an extended temperature range, 4-300K, showed that the parent compound, $\mathrm{GaSr}_{2} \mathrm{YCu}_{2} \mathrm{O}_{7}$, is weakly paramagnetic with no magnetic order above $4 \mathrm{~K}$.

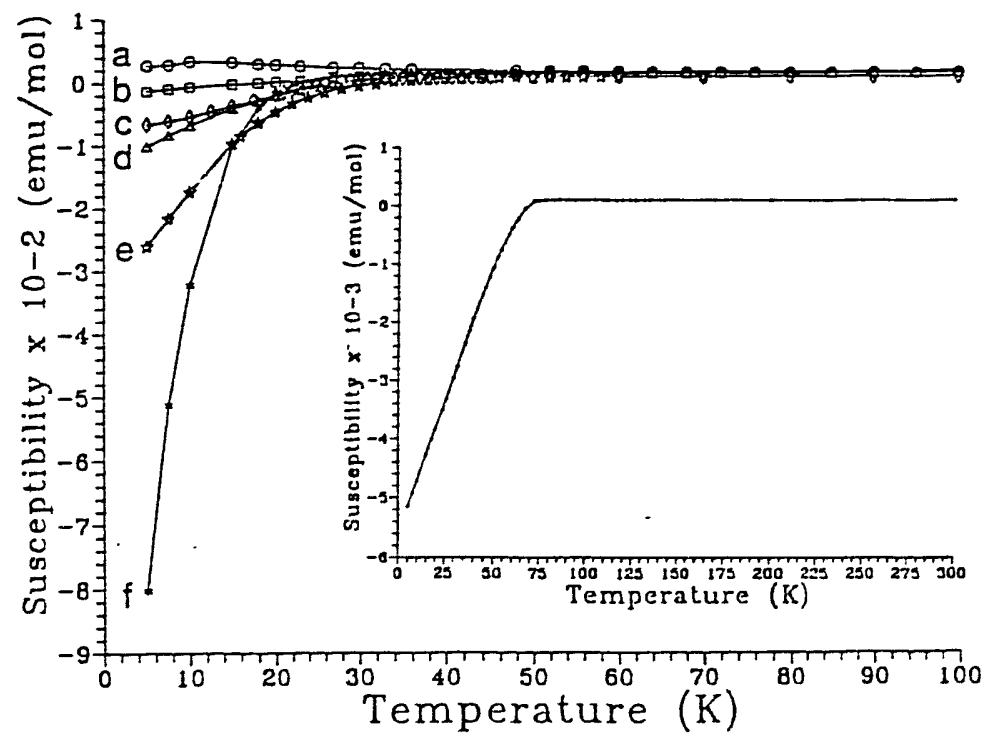

Fig.2 Squid susceptibility for dense $x=0$ (a), 0.1 (b), 0.15 (c), 0.2 (d), 0.25 (e) and 0.35 (f) samples annealed under $300 \mathrm{~atm}$. at $925^{\circ} \mathrm{C}$. Insert: susceptibility for $\mathrm{x}=0.35$ powder sample. 


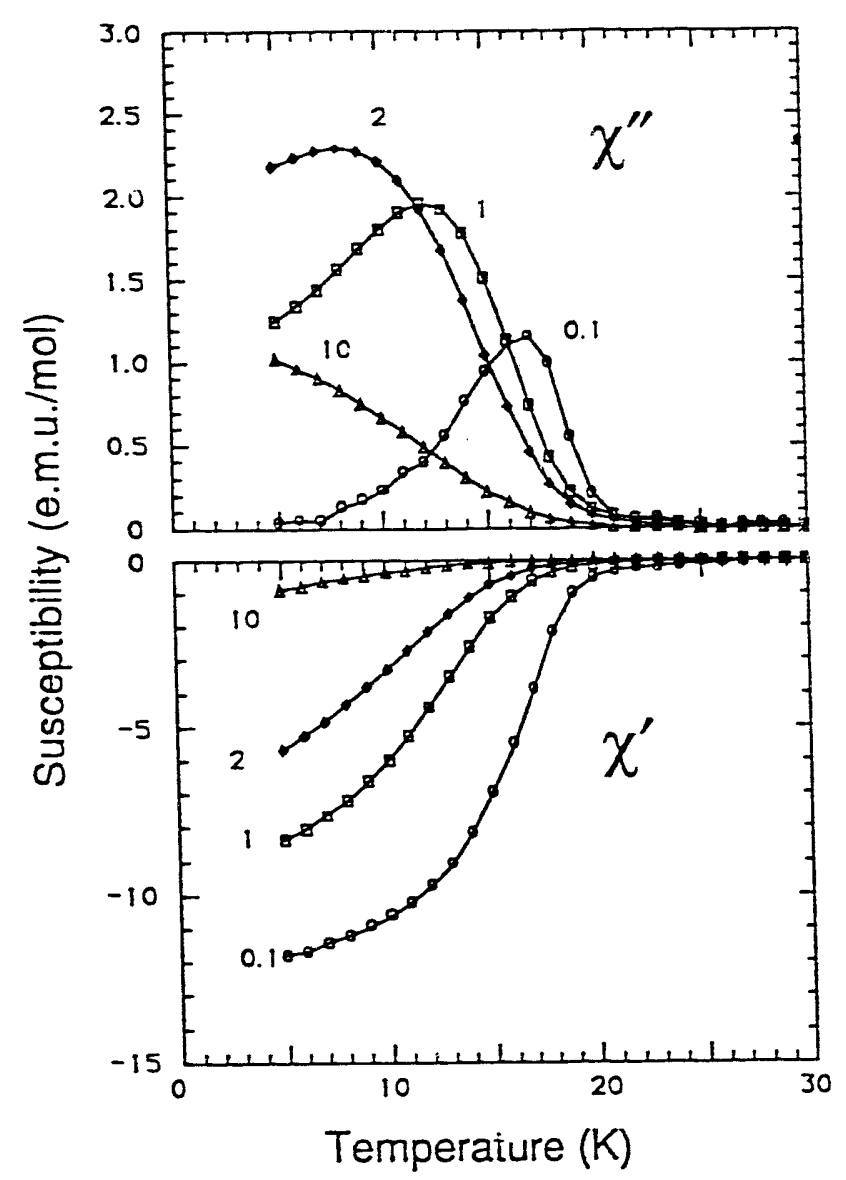

Fig.3 Real $\left(\chi^{\prime}\right)$ and imaginary $\left(\chi^{\prime \prime}\right)$ part of complex a.c. susceptibility for a $x=0.35$ dense sample. The magnitude of an a.c. field is denoted on the graph in Gauss.

Additional low field measurements were performed using a.c. susceptibility. Figure 3 shows real and imaginary susceptibility for a $x=0.35$ dense sample for various values of the a.c. field. Clearly, for low fields, $\leq 1$ Gauss, the sample shows full diamagnetic behavior, proving that for this composition a large fraction of the sample becomes superconducting. Similar a. $:$. field dependence of the measured superconducting phase fraction was observed for the other compositions for either dense pellets or loose powders. The a.c. data also showed very good agreement for the onset $T_{C}$ measured with the squid. 


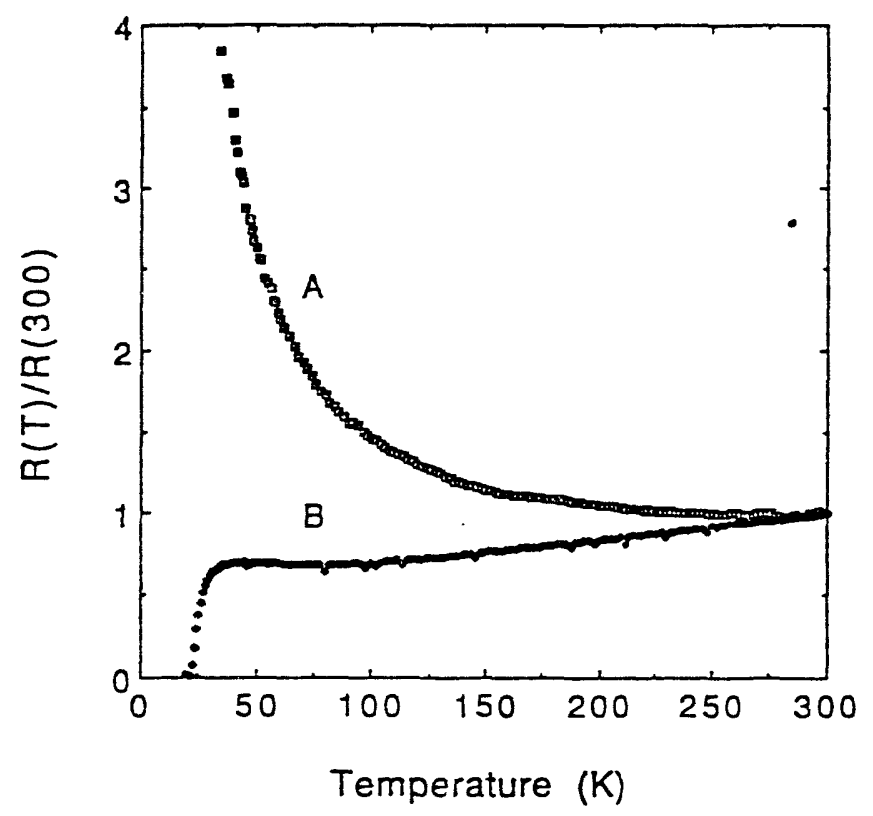

Fi.g. 4 Normalized resistance for the fast cooled (A) and high pressure annealed (B) dense samples.

Resistivity

measurements

confirmed superconductivity for the high pressure annealed material. Typical $R$ vs. $T$ data for the fast cooled (A) and high pressure oxygen annealed ( $B ; x=0.35$ dense samoles is shown on Fig.4. The resistance changes from semiconductor-like to metallic when the sample is annealed under increasingly oxidizing conditions. The almost linear dependence of resistance on temperature for the superconducting sample is the same as observed for all other high temperature superconductors.

\section{DISCUSSION}

To verify that the presence of gallium is a necessary condition for superconductivity, we have prepared several samples without $\mathrm{Ga}$ and processed them under the same conditions as the Ga-containing material. None of these samples showed any traces of either superconductivity or metallic behavior. Also, in the $x-$ ray diffraction patterns of $\mathrm{GaSr}_{2} \mathrm{Y}_{1-x} \mathrm{Ca}_{x} \mathrm{Cu}_{2} \mathrm{O}_{7}$ there was no indication for the formation of $\mathrm{YSI}_{2} \mathrm{Cu}_{3-\mathrm{x}} \mathrm{Ga}_{\mathrm{x}} \mathrm{O}_{7}$ impurity that could have superconducting properties. However, the reason the best superconducting properties are observed for $0.25 \leq x \leq 0.40$, i.e. beyond the apparent solubility limit at atmospheric pressure is still uncertain. It is possible, as frequently observed for 
oxide materials. that the solubility limit depends on the synthesis conditions [7]. Therefore, the solubility limit could extend beyond $x=0.25$ under high oxygen pressure annealing. The reduced impurity phase content after high pressure annealing indicates that some additional Ca might have been incorporated to the compound, however, the samples did not achieve full equilibrium during the 24 hour anneal. From thermogravimetric analysis there is a clear indication that the oxygen content increases slightly during cooling in oxygen over an extended temperature range for the doped compositions. Thus, the cooling rates are also very important and should be slow, $\angle 1^{\circ} \mathrm{C} / \mathrm{min}$.' for maximum oxygen uptake or diffusion of cations, especially for dense samples.

The strong dependence of the superconducting phase fraction on the magnitude of the a.c. field may be reiated to the expected large anisotropy of the critical magnetic fields. At present, this behavior is not fully understood, but a similar field dependence has been observed for other highly anisotropic copper oxide superconductors (e.g. $\left.\mathrm{Bi}_{2} \mathrm{Sr}_{2} \mathrm{CuO}_{6}\right)$ [8].

$x$-ray, neutron diffraction and thermogravimetric analysis, indicate that the undoped, $x=0$, material is stoichiometric in oxygen content and, thus, the copper ion should be in $\mathrm{d}^{9}$ configuration. The absence of antiferromagnetic order above $4 \mathrm{~K}$ in this material is significantly different from the parent compounds of other copper oxide superconductors in which the copper ion is unambiguously in a $d^{9}$ configuration, (e.g. $\mathrm{La}_{2} \mathrm{CuO}_{4}$ and $\mathrm{Nd}_{2} \mathrm{CuO}_{4}$ ). For these materials, the antiferromagnetic order develops at elevated temperatures $(2300 \mathrm{~K})$. In fact, the presence of antiferromagnetism in the parent material and superconductivity for doped compositions has led to several theories of high temperature superconductivity based on magnetic coupling[9]. Two-dimensional antiferromagnetic fluctuations are possible in $\mathrm{GaSr}_{2} \mathrm{YCu}_{2} \mathrm{O}_{7}$ but apparently the thick insulating, sroGaO-Sro, layer separating the double $\mathrm{CuO}_{2}$ planes inhibits magnetic coupling between the planes suppressing the development of three-dimensional antiferromagnetic order.

\section{CONCLUSION}

We have synthesized the first layered superconducting copper oxide with small, fixed oxidation state cations separating the conducting $\mathrm{AO}-\mathrm{CuO}_{2}-\mathrm{A}^{\prime}-\mathrm{CuO}_{2}-$ 
AO blocks, $\operatorname{GaSr}_{2} \mathrm{Y}_{1-\mathrm{x}} \mathrm{Ca}_{\mathbf{x}} \mathrm{Cu}_{2} \mathrm{O}_{7}$. This material may offer advantages for experimental and theoretical study because copper is the only mixed oxidation state ion and occurs only in one coordination. Several similar materials with ionic layers of $A 1 O$, NbO and $T a O$, may become superconducting once properly doped and annealed. These materials can provide important information concerning the nature of the superconducting state, in particular the relation between magnetic coupling and high temperature superconductivity.

This work is supported by the U.S. Depariment of Energy, Basic Energy Sciences / Materials sciences under contract No. W-31-109-ENG-38 (BD, AWM, DGH) and the National Science Foundation, Division of Materials Research - Low Temperature Physics Program under grant \#DMR 87-19738 (VZ) and the Science and Technology Center for superconductivity \#DMR-8821571 (PR, JTV, DAG, KRP).

\section{REFERENCES}

1. I.K. Schuller and J.D. Jorgensen, MRS Bulletin XIV, v.1, 27 (1989)

2. M.A. Beno, et al. Appl. Phys. Lett. 51, 57 (1987)

3. N. Murayama, et al. Jpn J. Appl. Phys. 2, 27 (1988)

4. M.T. Anderson and K.R. Poeppelmeier Chem. Mater. 3, 476 (1991)

5. J.T. Vaughey, et al. Chem. Mater. 3, 935 (1991)

6. G. Roth, et al. J. Phys. I 1, 721 (1991)

7. J.F. Bringley, et al. J. Solid State Chem. 88, 590 (1990)

8. R.M. Fleming, et al. Physica C 173, 37 (1990)

9. P.W. Anderson, Science 235, 1196 (1987) 

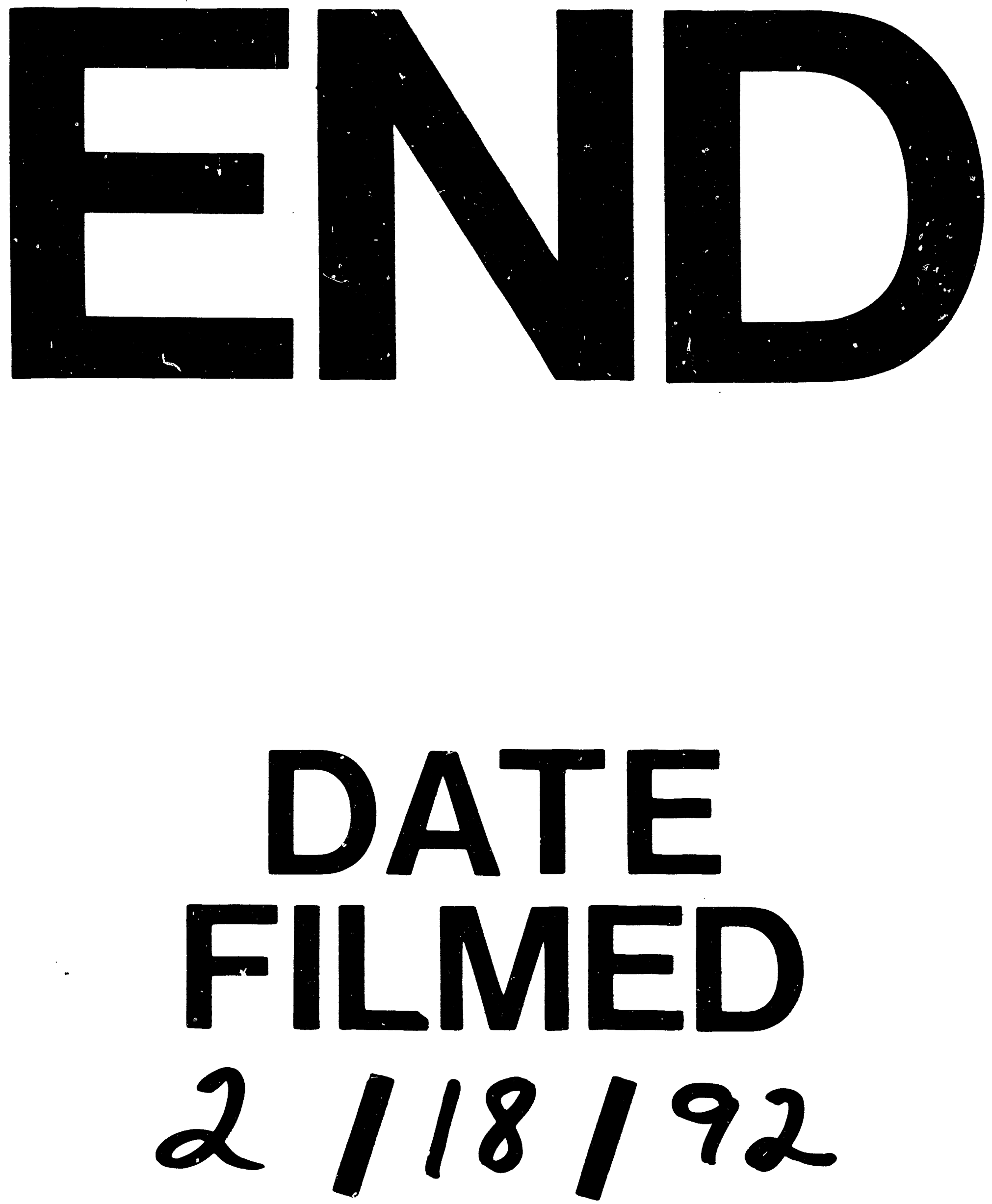
